\title{
Nivolumab in the treatment of advanced renal cell carcinoma
}

\author{
Address for correspondence: \\ Dr n. med. Piotr Tomczak \\ Szpital Kliniczny Przemienienia Pańskiego \\ Oddział Chemioterapii \\ ul. Szamarzewskiego 82/84, 60-568 Poznań \\ Phone: 618549038 \\ Fax: 618549072 \\ e-mail:md.piotr.tomczak@gmail.com
}

Oncology in Clinical Practice

2019, Vol. 15, No. 2, 124-126

DOI: $10.5603 / \mathrm{OCP} .2018 .0040$

Translation: eCORRECTOR

Copyright $(0) 2019$ Via Medica

ISSN 2450-1654

\begin{abstract}
Nivolumab is a programmed death receptor-1 (PD-1) blocking antibody approved for the treatment of advanced and metastatic renal cell carcinoma. Treatment with nivolumab is characterised by favourable toxicity profile. The occurrence of grade 3 and 4 toxicity during the therapy is low. This article describes a medical history of a patient with metastatic renal cell carcinoma treated with nivolumab.
\end{abstract}

Key words: renal cell carcinoma, immunotherapy, nivolumab

Oncol Clin Pract 2019; 15, 2: 124-126

\section{Introduction}

The occurrence rate of kidney cancer is about $2-3 \%$ of all human malignancies. In $90 \%$ of cases renal cell carcinoma (RCC) occurs [1]. The drugs used so far in the therapy of advanced renal cell carcinoma included tyrosine kinase inhibitors (TKI) and mTOR inhibitors (mammalian target of rapamycin inhibitors) [2].

Nivolumab is a monoclonal antibody that binds to the programmed cell death-1 receptor on the T lymphocyte and blocks its connection to the PD-L1 ligand present on cancer cells or other cell types present in the tumour microenvironment [3]. As a result of this inhibition, the activity of effector lymphocytes is not inhibited, which leads to the intensification of their cytotoxic effect on cancer cells. The CheckMate 025 study showed a $27 \%$ reduction in the hazard ratio (HR) of death (HR 0.73; p = 0.002) in the group of patients receiving nivolumab compared to patients treated with everolimus. Median overall survival (mOS) was 25 and 16. 9 months, respectively, in the studied groups [4]. In 2015 with accordance to the results of this study, the Food and Drug Administration (FDA) and the Euro- pean Commission registered nivolumab for treatment of advanced clear cell renal cell carcinoma (ccRCC) after failure of an earlier antiangiogenic therapy [5].

\section{Case report}

A 55-year-old man was admitted to the Department of Oncology in July 2015 with the diagnosis of stage IV kidney cancer. In June 2015 he underwent radical left-sided nephrectomy, and histopathological examination confirmed the diagnosis of ccRCC, G3, pT3b, pNx. At the time of admission to the hospital, the patient was in good general condition; his performance status (PS) was 1 in the Eastern Cooperative Oncology Group (ECOG) scale. He was treated for hypertension, type 2 diabetes mellitus, and Parkinson's disease. Complete blood count showed mild anaemia $(\mathrm{Hb} 7.8 \mathrm{mmol} / \mathrm{l})$. Prognosis of the patient in Memorial Sloan Kettering Cancer Centre scale (MSKCC) [6] was moderate. Computed tomography (CT) of the abdominal cavity revealed presence of a $48 \times 33 \mathrm{~mm}$ lymphadenopathy in the left paraaortic region, $22 \mathrm{~mm}$ 
foci in the right kidney, and thrombosis in the left renal vein stump. On the basis of CT imaging, an embolism in the LS10 artery was found. The patient received anticoagulant therapy and was qualified for first-line treatment with pazopanib.

The patient started the therapy on July 17, 2015. During the first 14 days of therapy, stage II inflammation of the oral mucosa was diagnosed according to common toxicity criteria for adverse events (CTCAE) v. 4.0. In addition, the patient reported reduced appetite, headaches, muscle aches, and increased blood pressure. During the next 14 days of treatment, blood pressure increased even more up to $220 / 110 \mathrm{~mm} \mathrm{Hg}$, and the patient required medical assistance in the hospital emergency department, where the treatment of hypertension was modified. Pazopanib therapy was stopped to normalise blood pressure, and it was decided to return to treatment at a reduced dose of $600 \mathrm{mg}$ once a day. However, due to persistently high blood pressure values, despite the intensification of hypotensive treatment, it was necessary to further reduce the dose of pazopanib to $400 \mathrm{mg}$ once daily. During further therapy, the patient remained in good condition and the blood pressure values remained normal.

According to Response Evaluation Criteria in Solid Tumours (RECIST) v. 1.1, in imaging studies performed after three months of treatment, the presence of partial response (PR) was noted, which lasted for the next six months. In May 2016 the progression of the disease in the form of numerous new metastases in the liver (the largest with a diameter of $17 \mathrm{~mm}$ ) and a metastatic focal point in the vertebral body L1 was found. The first line of treatment lasted for 11 months.

At the beginning of June 2016, according to the program of the National Health Fund (NFZ), the patient was qualified for a second line of treatment with axitinib. During the therapy, hoarseness and second-degree diarrhoea occurred. Due to coexisting arterial hypertension, increasing the drug dose was not possible, the patient continued therapy at a dose of $2 \times 5 \mathrm{mg} /$ day. At the first radiological assessment carried out in September 2016 progression of the disease was observed. There was an increase in metastases in the liver; the largest at that time had a diameter of $23 \mathrm{~mm}$. In addition, progression of the metastatic focus in the L1 vertebra with spinal muscle infiltration and tumour penetration into the spinal canal was observed. The second line of treatment was carried out for three months. Due to the pain, the patient was referred to lumbar radiotherapy and later received $4 \mathrm{mg}$ of zoledronic acid every four weeks. Since September 2016 he has not been treated systemically due to the lack of available therapeutic options in the third line of treatment.

In December 2016 the patient was qualified for treatment with nivolumab under the Early Access Programme. A CT examination performed before starting the patient on the third line of treatment showed further progression of metastatic lesions in the liver, with the largest diameter $42 \mathrm{~mm}$. In addition, the scan showed reduction of spinal muscle infiltration at $\mathrm{L} 1$ level, probably due to radiotherapy $(8 \mathrm{~Gy} / \mathrm{T})$. At the beginning of immunotherapy, the patient was in good general condition with well controlled blood pressure and sugar levels. Treatment with nivolumab was carried out at a dose of $3 \mathrm{mg} / \mathrm{kg}$ starting from January 4, 2017 and then from May 2018 at a constant dose of $240 \mathrm{mg}$ every two weeks [7].

During the treatment, first-degree diarrhoea episodes occurred and a first-degree creatinine level increase was observed but did not exceed that level. These did not require additional interventions [8]. In the CT examination performed after three months of treatment, metastases in the liver were reduced to $24 \mathrm{~mm}$ in the largest diameter, and in the next evaluation - to $10 \mathrm{~mm}$. Partial response to treatment (according to RECIST 1.1 criteria) has been maintained from June 2017 until now (October 2018). The patient has been continuing the treatment for 22 months, all the time with good tolerance and quality of life.

Table 1 presents the results of all lines of treatment.

Table 1. Course of treatment of the patient

\begin{tabular}{lccc}
\hline Line of treatment & I & II & III \\
\hline Drug & Pazopanib & Axitinib & PD \\
\hline Best response & PR & 3 & PR \\
\hline Treatment time, PFS (months) & Progression & Progression & Treatment is continued \\
\hline The cause of ending of the treatment & Yes & No, and without dose & increasing \\
\hline Treatment interruption & Yes (twice) & Yes & Does not apply \\
\hline Dose reduction & Yes & No \\
\hline Adverse effects & Yes & Good & Yes \\
\hline Adverse effects 3/4 & Quite good & No \\
\hline Quality of life & Very good
\end{tabular}




\section{Discussion}

Treating the vascular endothelial growth factor receptors (VEGFR) with tyrosine kinase inhibitors is associated with the occurrence of class-specific side effects such as arterial hypertension, hypothyroidism, inflammation of the oral mucosa, or chemotherapy-induced acral erythema [9]. In the course of immunocompetent treatment with PD-1 receptor inhibitors, a different profile of side effects is observed. Typical adverse reactions associated with anti-PD-1 treatment include pneumonia, nephritis, diarrhoea, and hypothyroidism, which require appropriate management depending on the severity [8].

In the CheckMate 025 studies, $79 \%$ of patients treated with nivolumab had adverse reactions of all degrees, compared to $88 \%$ treated with everolimus, while third- and fourth-degree adverse effects were observed in $19 \%$ vs. $37 \%$ of these patients, respectively. The quality of life in the group of patients receiving nivolumab was also better compared to the group of patients treated with everolimus [4]. In this case, it is worth noting that the patient remains in good general condition during immunotherapy, with minor side effects.

The benefit of nivolumab treatment in the CheckMate 025 study was reported in patients in all prognostic categories. Prognosis of patients was evaluated on the basis of the scale consisting of three factors (presence of anaemia, hypercalcaemia, and reduced efficiency). Patients without the above-mentioned factors were a group with favourable prognosis, with one unfavourable factor - a group with moderate prognosis, and finally with two or three factors - a group with poor prognosis. In this study, considering the group with favourable prognosis, the median overall survival (OS) for patients treated with nivolumab was not achieved compared to 19.6 months in patients treated with everolimus (HR $0.80)$. In the group with moderate prognosis the median OS was $21.8 v s .18 .4$ months (HR 0.81), and in the group with poor prognosis $15.3 \mathrm{vs} .7 .9$ (HR 0.48) [4, 10].

Benefit of treatment was also noted among patients with metastases to the bones and liver, i.e. those belonging to the group with worse prognosis. In patients with liver metastases the median OS was 18.3 vs. 16.0 months (HR 0.81), whereas in the group with bone metastases it was 18.5 vs. 13.8 months (HR 0.72). In the group of patients with pulmonary metastases better results were obtained - the median OS was 25 vs. 18.7 months (HR $0.72)[10]$

The percentage of objective response rate (ORR) in the group of patients treated with nivolumab was $25 \%$ compared to $5 \%$ in patients receiving everolimus; the dif- ference was statistically significant $(\mathrm{p}<0.001)$. A large proportion of the objective responses obtained in the CheckMate 025 study during treatment with nivolumab were permanent [4]. In the presented case, partial response with the third line of treatment was observed in a patient with moderate prognosis, with metastases to the bones and liver, which lasted for 16 months. The results of treatment with immunotherapy are better in the presented case in comparison to treatment with previous therapies in which tyrosine-kinase inhibitors were used.

\section{Conclusions}

Nivolumab is an anti-PD-1 drug with proven effectiveness in the treatment of patients with RCC and a favourable toxicity profile. In Poland it is currently available for use in the second line of treatment of advanced ccRCC and is a valuable therapeutic option in this indication.

\section{Reference}

1. Wysocki P, Borkowski T. Nowotwory układu moczowo-płciowego. In: Krzakowski M. ed. Onkologia kliniczna, t. 2. Via Medica, Gdańsk 2015: 751-760.

2. Escudier B, Porta C, Schmidinger M, et al. ESMO Guidelines Committee. Renal cell carcinoma: ESMO Clinical Practice Guidelines for diagnosis, treatment and follow-up. Ann Oncol. 2016; 27(Suppl 5): 58-68, doi: 10.1093/annonc/mdw328, indexed in Pubmed: 27664262.

3. Krawczyk P, Wojas-Krawczyk K. Immunoterapia ukierunkowana na immunologiczne punkty kontroli. In: Wysocki P. ed. Immunoonkologia. ViaMedica, Gdańsk 2016: 47-57.

4. Motzer R, Escudier B, McDermott D, et al. Nivolumab versus everolimus in advanced renal-cell carcinoma. N Engl J Med. 2015; 373(19): 1803-1813, doi: 10.1056/nejmoa1510665.

5. Xu JX, Maher VE, Zhang L, et al. FDA approval summary: nivolumab in advanced renal cell carcinoma after anti-angiogenic therapy and exploratory predictive biomarker analysis. Oncologist. 2017; 22(3): 311-317, doi: 10.1634/theoncologist.2016-0476, indexed in Pubmed: 28232599.

6. Motzer RJ, Mazumdar M, Bacik J, et al. Survival and prognostic stratification of 670 patients with advanced renal cell carcinoma. J Clin Oncol. 1999; 17(8): 2530-2540, doi: 10.1200/JCO.1999.17.8.2530, indexed in Pubmed: 10561319.

7. Zhao X, Suryawanshi S. Hruska M, et al. Assessment of nivolumab benefit-risk profile of a 240-mg flat dose relative to a 3-mg/kg dosing regimen in patients with advanced tumors. Ann Oncol. 2017; 28(8): 2002-2008, doi: 10.1093/annonc/mdx235, indexed in Pubmed: 28520840.

8. Brahmer J, Lacchetti C, Schneider B, et al. American Society of Clinical Oncology. Management of immune-related adverse events in patients treated with immune checkpoint inhibitor therapy: American Society of Clinical Oncology clinical practice guideline. J Clin Oncol. 2018; 36(17): 1714-1768, doi: 10.1200/jco.2017.77.6385.

9. Eisen T, Sternberg CN, Robert C, et al. Targeted therapies for renal cell carcinoma: review of adverse event management strategies. J Natl Cancer Inst. 2012; 104(2): 93-113, doi: 10.1093/jnci/djr511, indexed in Pubmed: 22235142.

10. Escudier B, Sharma P, McDermott D, et al. CheckMate 025 randomized phase 3 study: outcomes by key baseline factors and prior therapy for nivolumab versus everolimus in advanced renal cell carcinoma. Eur Urol. 2017; 72(6): 962-971, doi: 10.1016/j.eururo.2017.02.010. 